\title{
Orbital wall restoring surgery with resorbable mesh plate
}

Jae Doo Joo,

Dong Hee Kang,

Hyon Surk Kim

Department of Plastic and

Reconstructive Surgery, Dankook

University Hospital, Cheonan, Korea
Background: Orbital resorbable mesh plates are adequate to use for isolated floor and medial wall fractures with an intact bony buttress, but are not recommended to use for large orbital wall fractures that need load bearing support. The author previously reported an orbital wall restoring surgery that restored the orbital floor to its prior position through the transnasal approach and maintained temporary extraorbital support with a balloon in the maxillary sinus. Extraorbital support could reduce the load applied on the orbital implants in orbital wall restoring surgery and the use of resorbable implants was considered appropriate for the author's orbital wall restoring technique.

Methods: A retrospective review was conducted of 31 patients with pure unilateral orbital floor fractures between May 2014 and May 2018. The patients underwent transnasal restoration of the orbital floor through insertion of a resorbable mesh plate and maintenance of temporary balloon support. The surgical results were evaluated by the Hertel scale and a comparison of preoperative and postoperative orbital volume ratio (OVR) values.

Results: The OVR decreased significantly, by an average of $6.01 \%(p<0.05)$ and the preoperative and postoperative Hertel scale measurements decreased by an average of $0.34 \mathrm{~mm}$ with statistical significance $(p<0.05)$. No complications such as buckling or sagging of the implant occurred among the 31 patients.

Conclusion: The use of resorbable mesh plate in orbital floor restoration surgery is an effective and safe technique that can reduce implant deformation or complications deriving from the residual permanent implant.

Keywords: Orbital wall fractures / Resorbable mesh plate / Enophthalmos

\section{INTRODUCTION}

Autogenous materials are advantageous for use in orbital wall reconstruction in blowout fractures, but they have some problems such as donor site morbidity, unpredictable resorption rates, and increased operation time. Non-resorbable alloplastic

\section{Correspondence: Dong Hee Kang}

Department of Plastic and Reconstructive Surgery, Dankook University Hospital, 201 Manghyang-ro, Dongnam-gu, Cheonan 31116, Korea

E-mail: dhkcool@daum.net

${ }^{*}$ This article was presented at the PRS Korea 2017 on November 10-12, 2017 in Seoul, Korea

Received June 18, 2018 / Revised September 13, 2018 / Accepted September 17, 2018 implants (e.g., titanium or porous polyethylene) are widely used to avoid these drawbacks, but they also can cause late complications including infection, foreign body reaction, displacement and protrusion [1,2]. Recently, resorbable mesh plates have been increasingly used as implants in orbital wall reconstruction. Ideal resorbable orbital mesh plates should retain their mechanical strength over 1 to 2 years, allowing for adequate fibrous tissue formation on the bony orbital wall defect, and then be degraded and absorbed completely to minimize the risk of foreign body reaction [3]. The load-bearing strength of mesh plates is adequate for use in isolated floor and medial wall fractures with an intact bony buttress, but they are not recom- 
mended for use in large orbital wall fractures that need loadbearing support. Buckling and sagging of implants due to loss of their mechanical properties has been reported in large orbital wall fractures $[4,5]$, and there is a high possibility of late enophthalmos after total absorption; thus, the long-term results are questionable $[3,6]$.

The author previously reported an orbital wall restoring surgery that restored the orbital floor to its prior position through the transnasal approach and maintained temporary extraorbital support with a balloon in the maxillary sinus [7]. Previous studies, however, used nonabsorbable porous polyethylene implants. The bone defect of the orbital wall became smaller as the fractured orbital wall restored its prior position and the use of resorbable implants was expected to be reasonable in author's orbital wall restoring surgery technique. In addition, the temporary extraorbital support was expected to reduce the load applied on the orbital implants and also reduce postoperative buckling and sagging of resorbable implants in orbital wall restoration surgery. In this study, the author used the resorbable orbital mesh plate in orbital floor restoring surgery and reviewed the clinical outcomes.

\section{METHODS}

\section{Subjects}

The medical records of 42 patients who received surgical treatment for orbital floor fractures with a resorbable mesh plate between May 2014 and May 2018 were reviewed retrospectively. Patients were excluded if they showed any of the following criteria: orbital rim fracture, bilateral orbital fracture, and any history of facial fractures or diseases that could deform the orbital volume. A total of 31 patients underwent orbital wall restoring surgery with a resorbable mesh plate. The surgical indications for orbital wall fractures were (1) impairment of extraocular movement, (2) evidence of extensive fracture size (fracture size $>2 \mathrm{~cm}^{2}$ on computed tomography [CT] images), or (3) enophthalmos (>2 mm).

\section{Ophthalmic examination}

All surgical candidates underwent a preoperative examination by an ophthalmologist to assess diplopia and extraocular movement. The degree of enophthalmos was evaluated using Hertel exophthalmometer (Inami, Tokyo, Japan). The preoperative Hertel measurements were measured 1 day before surgery. The patients visited our outpatient clinic at 1 week, 1 month, 6 months, and 1 year after the operation. Hertel values were measured 6 months postoperatively after completion of orbital soft tissue atrophy and scar formation.

\section{CT scans and orbital volume measurements}

Three-dimensional CT images (GE Lightspeed VCT; GE Healthcare, Milwaukee, WI, USA), were taken in 2-mm-thick axial and 1-mm-thick coronal slices. CT scans were performed preoperatively and postoperatively during the outpatient follow-up period after at least 6 months. The Rapidia Image Postprocessing System (Infinitt, Seoul, Korea) was used to measure the volume of the orbits by tracing the orbital boundary on each CT image. The volume of the orbit on the untraumatized side was used as a control to eliminate differences in the individual orbital volume. The orbital volume ratio (OVR) was gained by dividing the volume of the traumatized orbit by that of the orbit on the normal side. The postoperative OVR was also measured in the same method.

\section{Surgical techniques}

Under general anesthesia, a transconjunctival incision with lateral canthotomy was used in the standard fashion. The fractured orbital wall and herniated orbital contents were exposed through the transorbital approach. After that, a curved Freer elevator was passed into the maxillary sinus through the maxillary ostium to restore the fractured orbital floor (Fig. 1A). The herniated orbital contents and the bony fragments were gently restored from the transnasal approach to their original position viewed via the transorbital site. A 16-Fr Foley catheter (Sewoon Medical, Seoul, Korea) was inserted through the same route with curved mosquito forceps (Fig. 1B) [7]. The Foley catheter balloon was inflated with an appropriate volume with physiologic saline solution, ranging from 12 to $15 \mathrm{~mL}$, until the anatomically restored orbit was maintained. A resorbable mesh plate (Rapidsorb; Synthes, West Chester, PA, USA) was molded to the proper size to cover the fractured orbital surface, and was inserted between the orbital contents and the orbital wall without any fixation (Fig. 1C, D) [7]. The balloon support was maintained in position for 7 days.

\section{Statistical analysis}

The measurements of the OVR and Hertel scale were analyzed using the paired $t$-test to identify statistically significant differences as a way of evaluating the surgical outcomes. The $p$-values $<0.05$ were considered to indicate statistical significance. All analyses were performed using IBM SPSS ver. 20.0 (IBM Corp., Armonk, NY, USA).

\section{RESULTS}

During the study period, 31 patients (24 males and seven females; age, 4-48 years; mean age, 21.6 years) with unilateral or- 

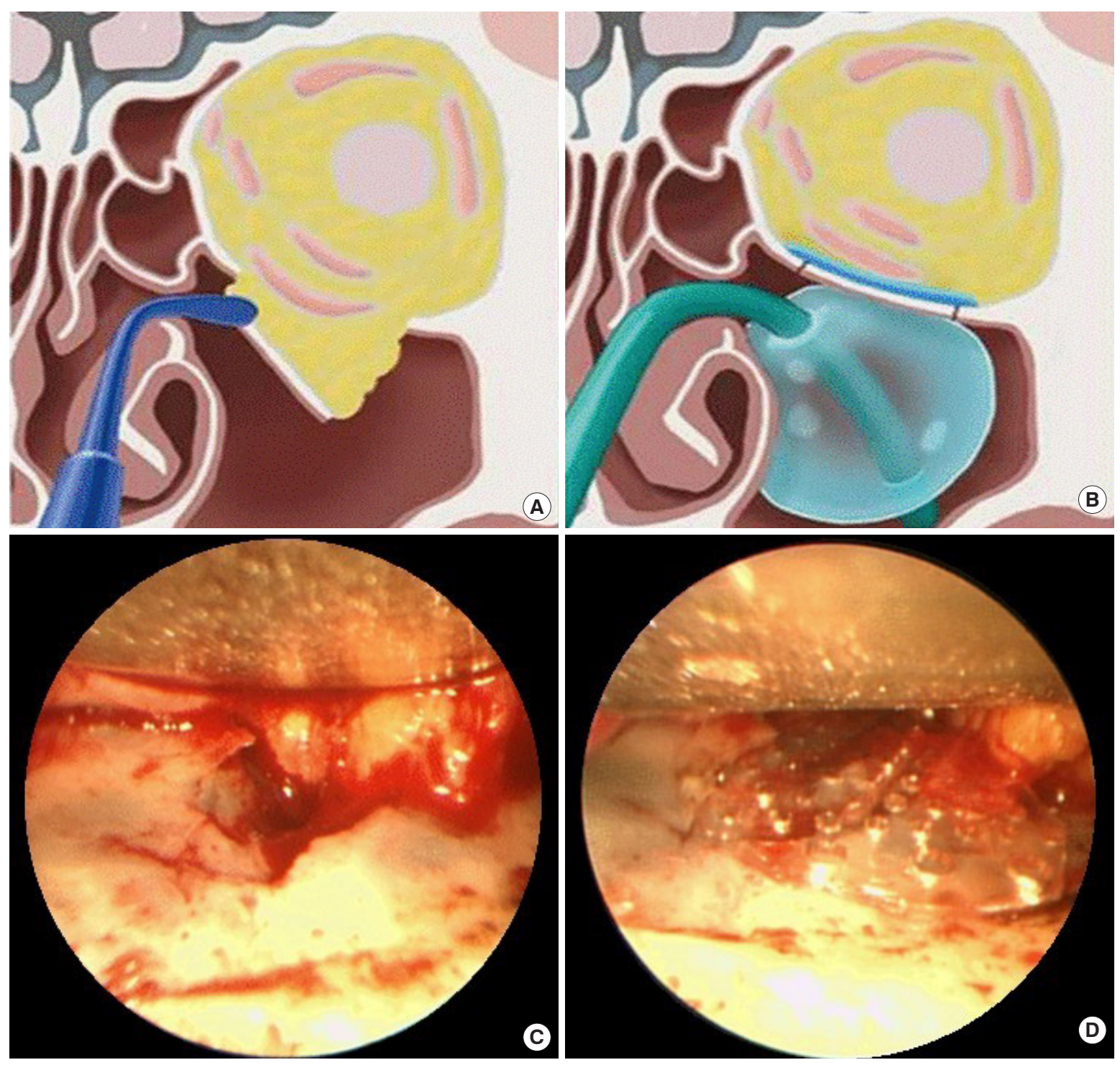

Fig. 1. Orbital wall restoration surgery with resorbable mesh plate. (A, B) Orbital wall restoration technique in an orbital floor fracture. The orbital floor was restored to its prior position with a curved Freer elevator, and temporary balloon support was maintained in the maxillary sinus. (C) The fractured orbital floor was exposed through a transconjunctival approach. (D) The orbital floor was restored and resorbable mesh plate was placed over the restored orbital floor. Adapted from Lim et al. Arch Plast Surg 2014;41:686-92 [7].

bital wall fractures underwent orbital restoration surgery using a resorbable mesh plate. The average surgical timing was 9.8 days after trauma (range, 2 to 28 days). The most common cause of injury was assault trauma (19 cases), followed by falling (six cases), traffic accidents (five cases), and occupational injury (one case). The mean follow-up period for the CT scans was 8.3 months after surgery (Figs. 2, 3). Before the surgery, four patients had enophthalmos, as defined by a Hertel measurement ( $>2 \mathrm{~mm}$ ). Sixteen patients had diplopia, and $14 \mathrm{pa}-$ tients had limited extraocular movement before the operation. Six of those patients showed muscle incarceration. Postoperatively, the ocular problems resolved after surgery in all patients, with no complications. In all 31 patients, postoperative buck- ling and sagging of resorbable implants were not observed on CT scans at 6 months postoperatively. The measurements on the Hertel scale increased by an average of $0.34 \mathrm{~mm}$ postoperatively. The changes in the Hertel scale measurements were statistically significant $(p<0.05)$. The mean preoperative OVR of the affected orbit was $108.39 \%$, while the mean postoperative OVR was $102.38 \%$. The difference in the OVR (6.01\%) was statistically significant $(p<0.05)$ (Table 1$)$.

\section{DISCUSSION}

The ideal implant should be biocompatible, sterilizable, easy to manipulate, and capable of reproducing the original orbit shape 




Fig. 2. A case of an 18-year-old female with a falling injury. (A, B) Preoperative and (C, D) 6-month postoperative coronal and sagittal section of computed tomography.
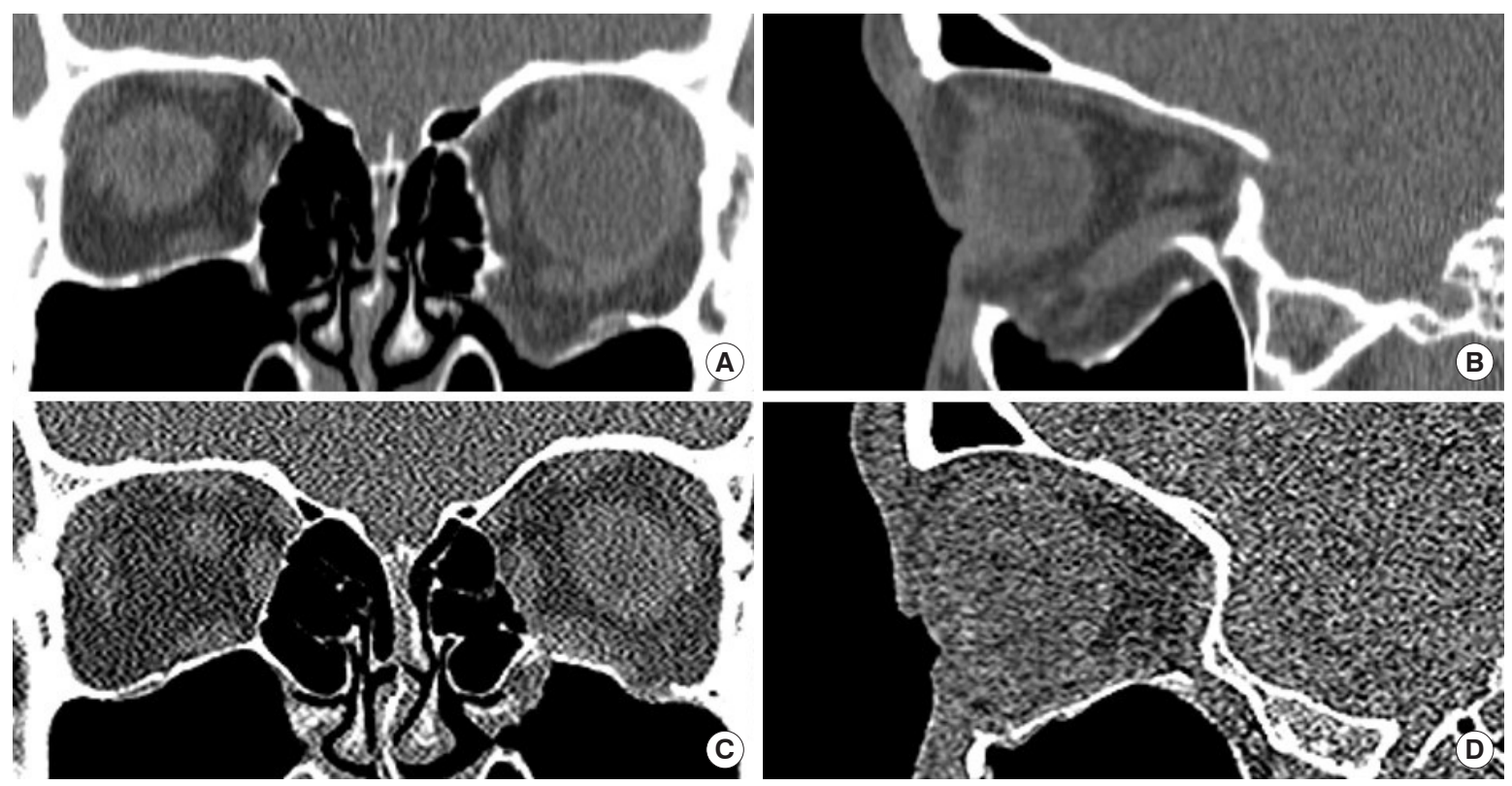

Fig. 3. A case of a 22-year-old male with trauma from assault. (A, B) Preoperative and (C, D) 6-month postoperative coronal and sagittal section of computed tomography.

Table 1. Preoperative and postoperative orbital volume ratio and Hertel scale

\begin{tabular}{lcccc}
\hline Variable & Preoperative & Postoperative & $\Delta$ Value & $p$-value \\
\hline Orbital volume ratio (\%) & $108.39 \pm 8.69$ & $102.38 \pm 4.48$ & $6.01^{\mathrm{a}} \pm 5.96$ & 0.001 \\
Hertel scale $(\mathrm{mm})$ & $-0.45 \pm 0.88$ & $-0.11 \pm 0.72$ & $0.34^{\mathrm{a})} \pm 0.85$ & 0.034 \\
\hline
\end{tabular}

Values are presented as mean \pm standard deviation.

$\Delta$, difference between preoperative and postoperative value.

a) Significant difference, $p<0.05$ in paired $t$-test.

[8]. Various materials have been introduced to reconstruct orbital wall fractures. Conventionally, autogenous bone, such as calvarial bone or iliac bone, is widely used. Autogenous bone has the advantage of a low risk of infection and host response, 
but it also has some problems, including donor site morbidity, unpredictable resorption rates, and increased operation time [9-11]. Currently, numerous alloplastic implants are produced and commonly used to reconstruct orbital wall fractures. These implants can avoid the drawbacks of the conventional method due to the shorter operative time, their ease of use, and the fact that they eliminate donor-site complications. However, especially in nonresorbable alloplastic implants (e.g., titanium or porous polyethylene), late complications can also occur, including infection, foreign body reaction, displacement, and protrusion [8]. The titanium mesh may lead to the adherence of orbital structures, resulting in extraocular movement restriction or eyelid retraction postoperatively [12]. Porous polyethylene remain as a foreign body, which may cause delayed-onset inflammation, despite its biocompatibility [1]. In contrast, the resorbable mesh plate, composed of polymers (polylactic acid and polyglycolic acid), is largely free from these disadvantages. Resorbable implant combines the advantages of conventional alloplastic implant with the advantage of being biodegradable in vivo. Few cases reported complications due to inflammatory reaction during resorption period, but the rates were $0 \%-2 \%$ [3]. The resorbable mesh plate retains its mechanical strength over 1 to 2 years, allowing for adequate fibrous tissue formation on the bony orbital wall defect, after which it is degraded and absorbed completely, minimizing the risk of foreign body reaction [3]. Therefore, it is useful in isolated floor or medial wall fractures with an intact bony buttress or minimal fracture site $[6,13,14]$. However, these implants can undergo sagging or buckling due to untimely loss of mechanical strength in large fractures $[4,5,15]$, and they show a late enophthalmos rate of $5 \%$ to $16 \%$ [3].

The author previously reported an orbital wall restoring surgery that restored the orbital floor to its prior position through the transnasal approach and maintained temporary extraorbital support with a balloon in the maxillary sinus [7]. This procedure significantly decreased the extent of the orbital bone defect and increased bony continuity due to the anatomical restoration from the transnasal approach. There is little risk of further volume change, since the primary orbital fragments heal in their original position. Furthermore, extraorbital ballooning temporarily provided counter-support to reduce the load applied on the implant. Thus, we expected that the resorbable mesh plate could maintain the restored bony orbit against the herniation load of the orbital contents, even in large orbital wall fractures, and temporary extraorbital support would prevent sagging and buckling of the orbital resorbable mesh plate.

To analysis our hypothesis, we used the OVR and the Hertel scale. The OVR is a useful parameter for evaluating orbital wall fractures perioperatively [16]. Our finding that the OVR decreased in long-term follow-up CT scans has convinced us that the orbital wall restoring surgery with the resorbable mesh plate is effective. However, this study has several limitations. First, it was a retrospective review with a limited sample size. Additionally, resorbable implants are well known to be resorbed within 12 months and lose their mechanical strength below the orbital threshold after about 1 to 6 months $[3,17]$. The follow-up period (mean follow-up period, 8.3 months) was relatively short compared to the total absorption time. However, we expected that there would be no significant difference after 6 months of bone maturation, due to the anatomical restoration of the orbital wall. Finally, we only studied isolated orbital floor fractures, so extended fractures, such as inferomedial wall fractures with bony strut involvement, were not enrolled. Further studies with a larger sample, longer prospective observation, and analysis of extended fractures would be useful supplements to this study.

In conclusion, the orbital wall restoring surgery with resorbable mesh plate is effective for restoring orbital volume and shape. The temporary extraorbital support is expected to reduce the load applied on the orbital implants and also reduce postoperative buckling and sagging of resorbable implants. The use of absorbable implants is a safe way to reduce implant deformation and complications from residual permanent implants in orbital wall restoration surgery.

\section{CONFLICT OF INTEREST}

No potential conflict of interest relevant to this article was reported.

\section{PATIENT CONSENT}

The patients or the parents of the patient provided written informed consent for the publication and the use of their images.

\section{ORCID}

$\begin{array}{ll}\text { Jae Doo Joo } & \text { https://orcid.org/0000-0003-1940-119X } \\ \text { Dong Hee Kang } & \text { https://orcid.org/0000-0002-6673-9655 } \\ \text { Hyon Surk Kim } & \text { https://orcid.org/0000-0001-6576-8035 }\end{array}$

\section{REFERENCES}

1. Aryasit O, Ng DS, Goh AS, Woo KI, Kim YD. Delayed onset porous polyethylene implant-related inflammation after orbital blowout fracture repair: four case reports. BMC Ophthalmol 2016;16:94. 
2. Yilmaz M, Vayvada H, Aydin E, Menderes A, Atabey A. Repair of fractures of the orbital floor with porous polyethylene implants. Br J Oral Maxillofac Surg 2007;45:640-4.

3. Ramesh S, Hubschman S, Goldberg R. Resorbable implants for orbital fractures: a systematic review. Ann Plast Surg 2018;81: 372-9.

4. Al-Sukhun J, Tornwall J, Lindqvist C, Kontio R. Bioresorbable poly-L/DL-lactide (P[L/DL]LA 70/30) plates are reliable for repairing large inferior orbital wall bony defects: a pilot study. J Oral Maxillofac Surg 2006;64:47-55.

5. Losken HW, van Aalst JA, Mooney MP, Godfrey VL, Burt T, Teotia S, et al. Biodegradation of Inion fast-absorbing biodegradable plates and screws. J Craniofac Surg 2008;19:748-56.

6. You JP, Kim DW, Jeon BJ, Jeong SH, Han SK, Dhong ES, et al. Two-year follow-up on the use of absorbable mesh plates in the treatment of medial orbital wall fractures. Arch Plast Surg 2013;40:728-34.

7. Lim NK, Kang DH, Oh SA, Gu JH. Orbital wall restoring surgery in pure blowout fractures. Arch Plast Surg 2014;41:68692.

8. Potter JK, Ellis E. Biomaterials for reconstruction of the internal orbit. J Oral Maxillofac Surg 2004;62:1280-97.

9. Converse JM, Smith B, Obear MF, Wood-Smith D. Orbital blowout fractures: a ten-year survey. Plast Reconstr Surg 1967; 39:20-36.

10. Smith JD, Abramson M. Membranous vs endochondrial bone autografts. Arch Otolaryngol 1974;99:203-5.
11. Lin KY, Bartlett SP, Yaremchuk MJ, Fallon M, Grossman RF, Whitaker LA. The effect of rigid fixation on the survival of onlay bone grafts: an experimental study. Plast Reconstr Surg 1990;86:449-56.

12. Lee HB, Nunery WR. Orbital adherence syndrome secondary to titanium implant material. Ophthalmic Plast Reconstr Surg 2009;25:33-6.

13. Kim YJ, Choi SH, Jun YJ, Seo BC. Open reduction in trapdoortype blowout fractures using absorbable mesh plates. J Craniofac Surg 2011;22:2263-7.

14. Hwang WJ, Lee DH, Choi W, Hwang JH, Kim KS, Lee SY. Analysis of orbital volume measurements following reduction and internal fixation using absorbable mesh plates and screws for patients with orbital floor blowout fractures. J Craniofac Surg 2017;28:1664-9.

15. Hwang K, Kim DH. Comparison of the supporting strength of a poly-L-lactic acid sheet and porous polyethylene (Medpor) for the reconstruction of orbital floor fractures. J Craniofac Surg 2010;21:847-53.

16. Oh SA, Aum JH, Kang DH, Gu JH. Change of the orbital volume ratio in pure blow-out fractures depending on fracture location. J Craniofac Surg 2013;24:1083-7.

17. Miller RA, Brady JM, Cutright DE. Degradation rates of oral resorbable implants (polylactates and polyglycolates): rate modification with changes in PLA/PGA copolymer ratios. J Biomed Mater Res 1977;11:711-9. 